\title{
Nuclear factor IB is downregulated in vulvar squamous cell carcinoma (VSCC): Unravelling differentially expressed genes in VSCC through gene expression dataset analysis
}

\author{
SHATAVISHA DASGUPTA ${ }^{1}$, PATRICIA C. EWING-GRAHAM ${ }^{1}$, THIERRY P.P. VAN DEN BOSCH ${ }^{1}$, \\ SIGRID M.A. SWAGEMAKERS ${ }^{1,2}$, LINDY A.M. SANTEGOETS ${ }^{3}$, HELENA C. VAN DOORN ${ }^{4}$, \\ PETER J. VAN DER SPEK ${ }^{1,2}$, SENADA KOLJENOVIĆ ${ }^{1}$ and FOLKERT J. VAN KEMENADE ${ }^{1}$
}

\author{
Departments of ${ }^{1}$ Pathology and ${ }^{2}$ Clinical Bioinformatics, Erasmus MC, University Medical Center Rotterdam, \\ 3000CA Rotterdam; ${ }^{3}$ Department of Obstetrics and Gynecology, Reinier de Graaf Gasthuis, \\ 2625 AD Delft; ${ }^{4}$ Department of Gynecologic Oncology, Erasmus MC Cancer Institute, \\ University Medical Center Rotterdam, 3000CA Rotterdam, The Netherlands
}

Received November 19, 2020; Accepted February 12, 2021

DOI: $10.3892 / \mathrm{ol} .2021 .12642$

\begin{abstract}
Vulvar squamous cell carcinoma (VSCC) comprises two distinct etiopathological subtypes: i) Human papilloma virus (HPV)-related VSCC, which arises via the precursor high grade squamous intraepithelial lesion (HSIL); and ii) HPV-independent VSCC, which arises via precursor, differentiated vulvar intraepithelial neoplasia (dVIN), driven by TP53 mutations. However, the mechanism of carcinogenesis of VSCC is poorly understood. The current study aimed to gain insight into VSCC carcinogenesis by identifying differentially expressed genes (DEGs) for each VSCC subtype. The expression of certain DEGs was then further assessed by performing immunohistochemistry (IHC) on whole tissue sections of VSCC and its precursors. Statistical analysis of microarrays was performed on two independent gene expression datasets (GSE38228 and a study from Erasmus MC) on VSCC and normal vulva. DEGs were identified that were similarly (up/down) regulated with statistical significance in both datasets. For HPV-related VSCCs, this constituted 88 DEGs, and for HPV-independent VSCCs, this comprised 46 DEGs. IHC was performed on VSCC $(n=11)$, dVIN $(n=6)$, HSIL ( $n=6)$ and normal vulvar tissue ( $n=7$ ) with i) signal transducer and activator of transcription 1 (STAT1; an upregulated DEGs); ii) nuclear factor IB (NFIB; a downregulated DEG); iii) p16 (to determine the HPV status of tissues); and iv) p53 (to confirm the histological diagnoses). Strong and diffuse
\end{abstract}

Correspondence to: Dr Shatavisha Dasgupta, Department of Pathology, Erasmus MC, University Medical Center Rotterdam, Postbus 2040, Be-building, 3000CA Rotterdam, The Netherlands E-mail: s.dasgupta@erasmusmc.nl

Key words: vulva, female genital tract, gene expression profiling, gene expression omnibus database, differentially expressed genes, immunohistochemistry, bioinformatics, nuclear transcription factors
NFIB expression was observed in the basal and para-basal layers of normal vulvar tissue, whereas NFIB expression was minimal or completely negative in dVIN and in both subtypes of VSCC. In contrast, no discernable difference was observed in STAT1 expression among normal vulvar tissue, dVIN, HSIL or VSCC. By leveraging bioinformatics, the current study identified DEGs that can facilitate research into VSCC carcinogenesis. The results suggested that NFIB is downregulated in VSCC and its relevance as a diagnostic/prognostic biomarker deserves further exploration.

\section{Introduction}

Vulvar squamous cell carcinoma (VSCC) constitutes $90 \%$ of all vulvar malignancies, and its incidence has risen over the past decades $(1,2)$. Approximately $25 \%$ of VSCCs arise in association with a human papillomavirus (HPV)-infection, via the precursor lesion, high grade squamous intraepithelial lesion (HSIL) (3). The majority (75\%) of VSCCs, however, is postulated to develop on the background of chronic dermatoses, via the precursor lesion, differentiated vulvar intraepithelial neoplasia (dVIN) (3).

The dual pathogenesis of VSCC has been recognized several years ago, however, molecular mechanisms of the carcinogenesis have not been well characterized (4). This is largely because the genomic profiles of VSCC or its precursor lesions have been investigated in only a few studies so far (1,5-11). These studies identified somatic mutations of TP53 to be the pivotal oncogenic driver of HPV-independent VSCC, and also detected genomic alterations of PIK3CA, HRAS, or FGFR 3 in both subtypes of VSCC $(7-9,11)$. Nevertheless, limited sample sizes and dissimilar methodologies of these studies have prevented significant advancement of knowledge of VSCC carcinogenesis (4).

A better understanding of the molecular pathways involved in VSCC carcinogenesis can enable identification of biomarkers that may be used to improve the diagnosis, for prognostic stratification, or as targets for precision treatment. Currently, 
the mainstay of VSCC treatment is surgical excision, which is often associated with post-operative morbidities due to the anatomical complexity of the vulvar region. Discovering novel biomarkers for targeted treatment may help improve personalization of treatment for patients with VSCC.

A key method for discovering candidate biomarkers is through identifying genes that are differentially expressed in cancer tissue and normal tissue (12). To this end, we analyzed datasets of gene expression microarray on VSCC and normal vulvar tissue, from two independent studies, using the latest bioinformatics tools. We further investigated the expression of some of the differentially expressed genes (DEGs) identified thereby, by performing immunohistochemistry (IHC) on VSCC, HSIL, dVIN, and normal vulvar tissue.

\section{Materials and methods}

Identification and analysis of datasets. A publicly available dataset (GSE38228) was identified and downloaded from gene expression omnibus (GEO) (13). This dataset consists of VSCCs $(n=14)$ and normal vulvar tissues $(n=5)$, for which gene expression microarray had been performed using the gene-chip platform Illumina HumanHT-12 V4.0. A 2nd dataset was obtained from a study previously conducted by researchers at our center. This dataset consists of VSCCs $(n=5)$, for which gene expression microarray was performed using the gene-chip platform Affymetrix HG U133 Plus 2.0.

The datasets were imported into OmniViz (version 6.1.13.0, BioWisdomLtd.). Statistical analysis of microarrays (SAM) was performed to identify DEGs using the following cutoff-values-a false discovery rate (FDR) of $\leq 0.01$ and a fold change of 1.5 . P-value $<0.05$ was considered as statistically significant. Functional annotations of the SAM results were done using Ingenuity Pathway Analysis (IPA, Qiagen, Inc.). Expression levels of p16 (CDKN2A), which is known to be overexpressed in HPV-related VSCC, were used to distinguish the samples as HPV-related or HPV-independent VSCC. For both subtypes of VSCC, DEGs that were upregulated or downregulated in both datasets with statistical significance were identified. The Database for Annotation, Visualization and Integrated Discovery (DAVID; version 6.8) was used to identify the most significantly enriched functional genes $(14,15)$. Gene ontology (GO) enrichment analyses were performed using the DAVID online tool to annotate biological process, cellular component, and molecular function of DEGs. Additional information on the DEGs was obtained from IPA, cBioPortal, and Gene Expression Profiling Interactive Analysis (GEPIA). Protein-protein interaction (PPI) networks of the DEGs were constructed using Search Tool for the Retrieval of Interacting Genes (STRING) (16-19).

Immunohistochemistry (IHC). Formalin fixed paraffin embedded (FFPE)-tissues of VSCC, HSIL, dVIN, and normal vulva were retrieved from the archives of Department of Pathology, Erasmus MC. Histology of all tissues was reviewed by two pathologists (SDG and PCEG). Patient data were anonymized and patient materials were handled following the guidelines of World Medical Association Declaration of Helsinki.
For performing IHC, DEGs were selected-i) that were expressed in the cytoplasm or nucleus and ii) for which primary antibodies were commercially available. In addition, for all samples, IHC was performed with p16 to determine the HPV-status, and with 553 to confirm the histological diagnoses. Sequential sections of $4 \mu \mathrm{m}$-thickness were prepared from the FFPE-tissues and automated IHC was performed using the Ventana Benchmark ULTRA (Ventana Medical Systems Inc.), following the manufacturer's instructions (Data S1 and Table SI).

The IHC markers were scored as follows: For the IHC markers of DEGs, the percentage of cells showing staining, irrespective of the intensity of staining, was assessed manually. In addition, the intensity of staining (weak, moderate, and strong) and the distribution of staining within the epithelium was recorded. p16-expression patterns were scored as block-type or non-block-type (patchy), following the guidelines of Lower Anogenital Squamous Terminology Standardization Project (LAST) (16). Block-type p16-expression, i.e. diffuse, continuous, moderate-to-intense nuclear and/or cytoplasmic staining in $\geq 1 / 3$ rd of the epithelial thickness is considered to be a reliable surrogate marker of high-risk HPV-infection (20). p53-expression patterns were scored as p53-mutant or p53-wild-type following descriptions in recent literature $(10,21)$. p53-mutant patterns have been reported to accurately reflect the presence of TP53 mutations (10). p53-mutant patterns include basal to para-basal/diffuse overexpression, basal overexpression, or aberrant negative/null-pattern. p53-wild-type patterns include scattered heterogeneous basal and/or para-basal expression, and scattered mid-epithelial expression with basal sparing. The latter p53-wild-type pattern is associated with HPV-related lesions $(10,22)$.

Ethics statement. This study was conducted in accordance with the guidelines of the Dutch Federation of Biomedical Scientific Societies (www.federa.org/codes-conduct), which state that no separate ethical approval is required for the use of anonymized residual tissue procured during regular treatment.

\section{Results}

Dataset analyses. From GSE38228, 3 samples were identified as HPV-related VSCC and 3 samples were identified as HPV-independent VSCC. A total of 342 genes (244 upregulated and 98 downregulated) were found to be differentially expressed with statistical significance only in HPV-related VSCC. A total of 382 genes (203 upregulated and 179 downregulated) were found to be differentially expressed with statistical significance only in HPV-independent VSCC. From the 2nd dataset, 3 samples were identified as HPV-related VSCC and 2 samples as HPV-independent VSCC. A total of 7005 genes were differentially expressed with statistical significance in HPV-related VSCC, and 4,283 genes were differentially expressed with statistical significance in HPV-independent VSCC.

Combining both datasets, for HPV-related VSCC, 88 DEGs were identified that were similarly regulated with statistical significance. This comprised 69 upregulated and 19 downregulated DEGs; signal transducer and activator of transcription 1 (STAT1) was one of the upregulated DEGs. For 


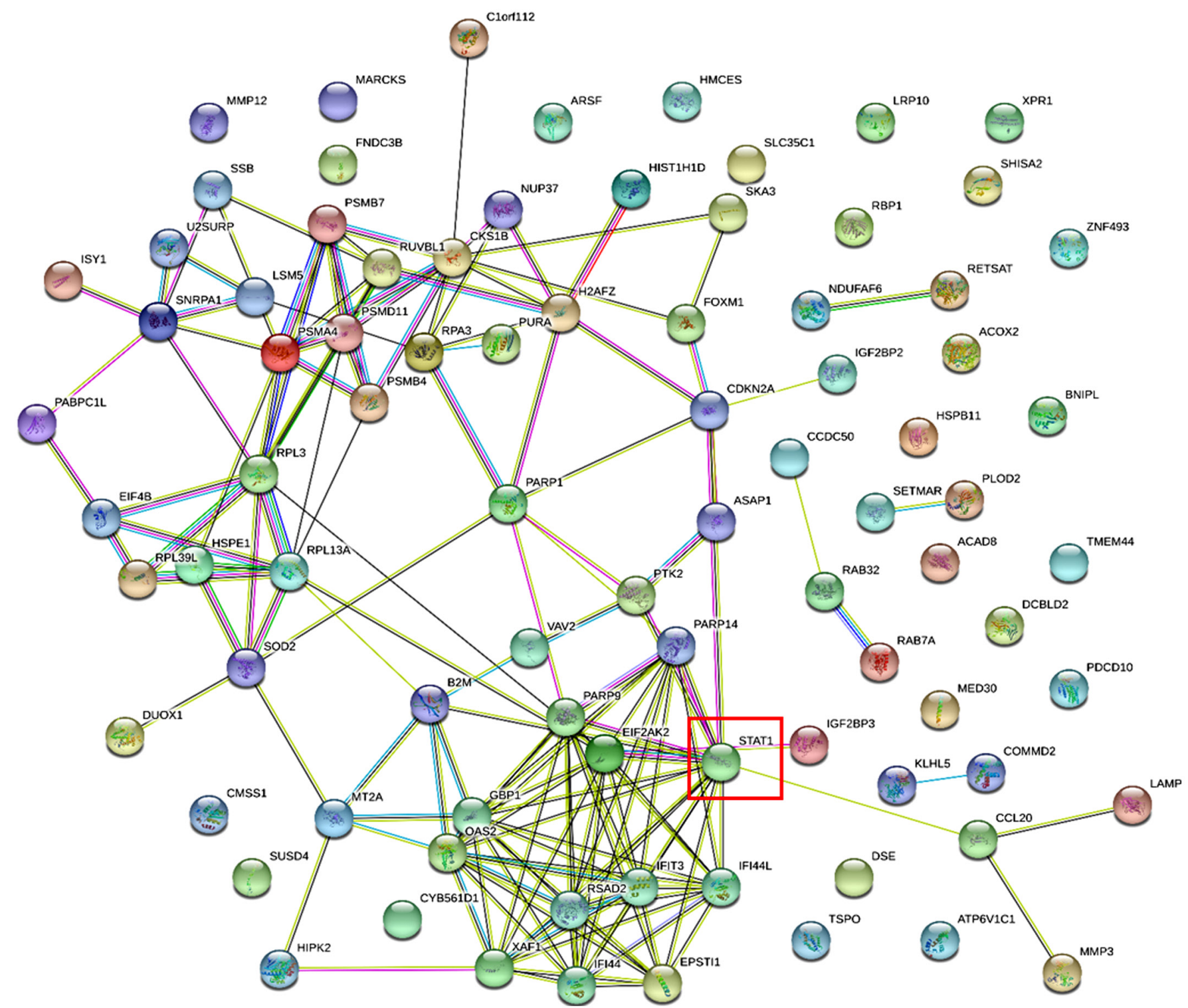

Figure 1. Protein-protein interaction network of the 88 differentially expressed genes in human papilloma virus-related vulvar squamous cell carcinoma (constructed with STRING). Signal transducer and activator of transcription 1 is indicated with a red box. The network nodes represent the proteins produced by a single, protein-coding gene locus. Colored nodes represent query proteins and first shells of interactions. White nodes represent second shell of interactions. Empty nodes represent proteins of an unknown 3D structure. Filled nodes represent proteins of which some 3D structures are known or predicted. The edges are coded as follows: Light blue, known interaction curated from databases; pink, known interaction determined through experiments; green, predicted interaction in the gene neighborhood; red, gene fusions; dark blue, gene co-occurrence; lime green, text mining; black, co-expression; indigo, protein homology.

HPV-independent VSCC, 46 DEGs were identified that were similarly regulated with statistical significance. This comprised 16 upregulated and 30 downregulated DEGs; nuclear factor IB (NFIB) was one of the downregulated DEGs. The PPI networks of these DEGs are visualized in Figs. 1 and 2, and the DEGs along with their subcellular locations, functions, and related canonical pathways are listed in Tables SII and SIII.

The DEGs identified for HPV-related VSCC mainly participate in response to stimulus and regulation of cellular and biological processes (Table I). As for the molecular function, these DEGs are mainly involved in binding with ions or signaling receptors (Table II). The cellular component of these DEGs include cytoplasm and extracellular region.

The DEGs identified for HPV-independent VSCC mainly participate in regulation of cellular and metabolic processes
(Table III). As for the molecular function, these DEGs are mainly involved in protein and ion binding (Table IV). The cellular component of these DEGs include membrane-bound organelles and the cytoplasm.

Immunohistochemistry. Primary antibodies for performing IHC were commercially available for i) STAT1, one of the upregulated DEGs, and ii) NFIB, one of the downregulated DEGs. IHC was performed on 11 VSCCs, 6 dVINs, 6 HSILs, and 7 normal vulva tissues; these were from women with a median age of 72.5 years (range, 26-90 years). Immunohistochemical expression of p53, p16, NFIB, and STAT1 are presented in Tables V and VI. For NFIB and STAT1, the IHC patterns observed in the tissues are described below, and the distribution of expression is depicted in Fig. S1. 
Table I. GO enrichment analysis of the differentially expressed genes for human papilloma virus-related vulvar squamous cell carcinoma.

A, Biological processes

\begin{tabular}{llll}
\hline Term & \multicolumn{1}{c}{ Description } & Gene count & P-value \\
\hline GO:0050896 & Response to stimulus & 35 & $2.89 \times 10^{-10}$ \\
GO:0050794 & Regulation of cellular process & 34 & $2.92 \times 10^{-05}$ \\
GO:0050789 & Regulation of biological process & 34 & $1.19 \times 10^{-04}$ \\
GO:0032501 & Multicellular organismal process & 33 & $6.85 \times 10^{-11}$ \\
GO:0007275 & Multicellular organism development & 26 & $1.39 \times 10^{-08}$ \\
GO:0048856 & Anatomical structure development & 26 & $7.60 \times 10^{-08}$ \\
GO:0042221 & Response to chemical & 25 & $8.56 \times 10^{-09}$ \\
GO:0007154 & Cell communication & 24 & $1.09 \times 10^{-05}$ \\
GO:0006950 & Response to stress & 23 & $8.86 \times 10^{-09}$ \\
GO:0007165 & Signal transduction & 23 & $6.01 \times 10^{-06}$ \\
\hline
\end{tabular}

B, Molecular functions

\begin{tabular}{llrr}
\hline Term & \multicolumn{1}{c}{ Description } & Gene count & P-value \\
\hline GO:0043167 & Ion binding & 29 & $2.59 \times 10^{-08}$ \\
GO:0005102 & Signaling receptor binding & 21 & $2.35 \times 10^{-13}$ \\
GO:0097367 & Carbohydrate derivative binding & 19 & $4.81 \times 10^{-09}$ \\
GO:0043169 & Integrin binding & 19 & $1.11 \times 10^{-04}$ \\
GO:0098772 & Molecular function regulator & 18 & $6.66 \times 10^{-05}$ \\
GO:0008201 & Heparin binding & 15 & $1.60 \times 10^{-21}$ \\
GO: 1901681 & Sulfur compound binding & 15 & $9.93 \times 10^{-19}$ \\
GO:0030545 & Receptor regulator activity & 12 & $9.24 \times 10^{-10}$ \\
GO:0005198 & Structural molecule activity & 9 & $5.41 \times 10^{-06}$ \\
GO:0005509 & Growth factor binding & $8.60 \times 10^{-05}$ \\
\hline
\end{tabular}

C, Cellular component

\begin{tabular}{llrr}
\hline Term & \multicolumn{1}{c}{ Description } & Gene count & P-value \\
\hline GO:0005737 & Cytoplasm & 33 & $4.88 \times 10^{-04}$ \\
GO:0005576 & Extracellular region & 30 & $1.54 \times 10^{-13}$ \\
GO:0012505 & Endomembrane system & 24 & $1.73 \times 10^{-07}$ \\
GO:0031982 & Vesicle & 20 & $5.59 \times 10^{-06}$ \\
GO:0043230 & Extracellular organelle & 14 & $1.66 \times 10^{-05}$ \\
GO:0031410 & Cytoplasmic vesicle & 14 & $8.38 \times 10^{-05}$ \\
GO:0097708 & Intracellular vesicle & 14 & $8.57 \times 10^{-05}$ \\
GO:0062023 & Collagen-containing extracellular Matrix & 13 & $6.10 \times 10^{-13}$ \\
GO:0070062 & Extracellular exosome & 13 & $7.18 \times 10^{-05}$ \\
GO: 1903561 & Extracellular vesicle & 13 & $7.96 \times 10^{-05}$ \\
\hline
\end{tabular}

GO, Gene Ontology.

STAT1. Normal vulvar tissue $(n=7)$ : Five showed diffuse, cytoplasmic STAT1-expression of moderate-to-strong intensity, across full epithelial thickness; two showed focal STAT-1 expression of moderate-to-strong intensity.

dVIN ( $n=6)$, HSIL $(n=6)$, HPV-related VSCC $(n=5)$, HPV-independent VSCC $(n=6)$ : All showed diffuse, cytoplasmic STAT1-expression of moderate-to-strong intensity, across full epithelial thickness.

NFIB. Normal vulvar tissue $(\mathrm{n}=7)$ : All showed strong, diffuse, nuclear NFIB-expression, predominantly along the basal layers, which occasionally extended to the para-basal layers. 
Table II. Functional annotation analysis of the differentially expressed genes for human papilloma virus-related vulvar squamous cell carcinoma.

Functional annotation cluster

Immunity

Antiviral defense

Defense response to virus

Host-virus interaction

Protease

Type I interferon signaling

Innate immunity

Perinuclear region of cytoplasm

Antigen processing and presentation of exogenous peptide antigen via MHC class I,

TAP-dependent

Tumor necrosis factor-mediated signaling
Gene count

P-value

$5 \times 10^{-06}$

$9 \times 10^{-09}$

$2 \times 10^{-07}$

$2 \times 10^{-05}$

$8 \times 10^{-05}$

$3 \times 10^{-07}$

$2 \times 10^{-05}$

$3 \times 10^{-05}$

$2 \times 10^{-07}$

5

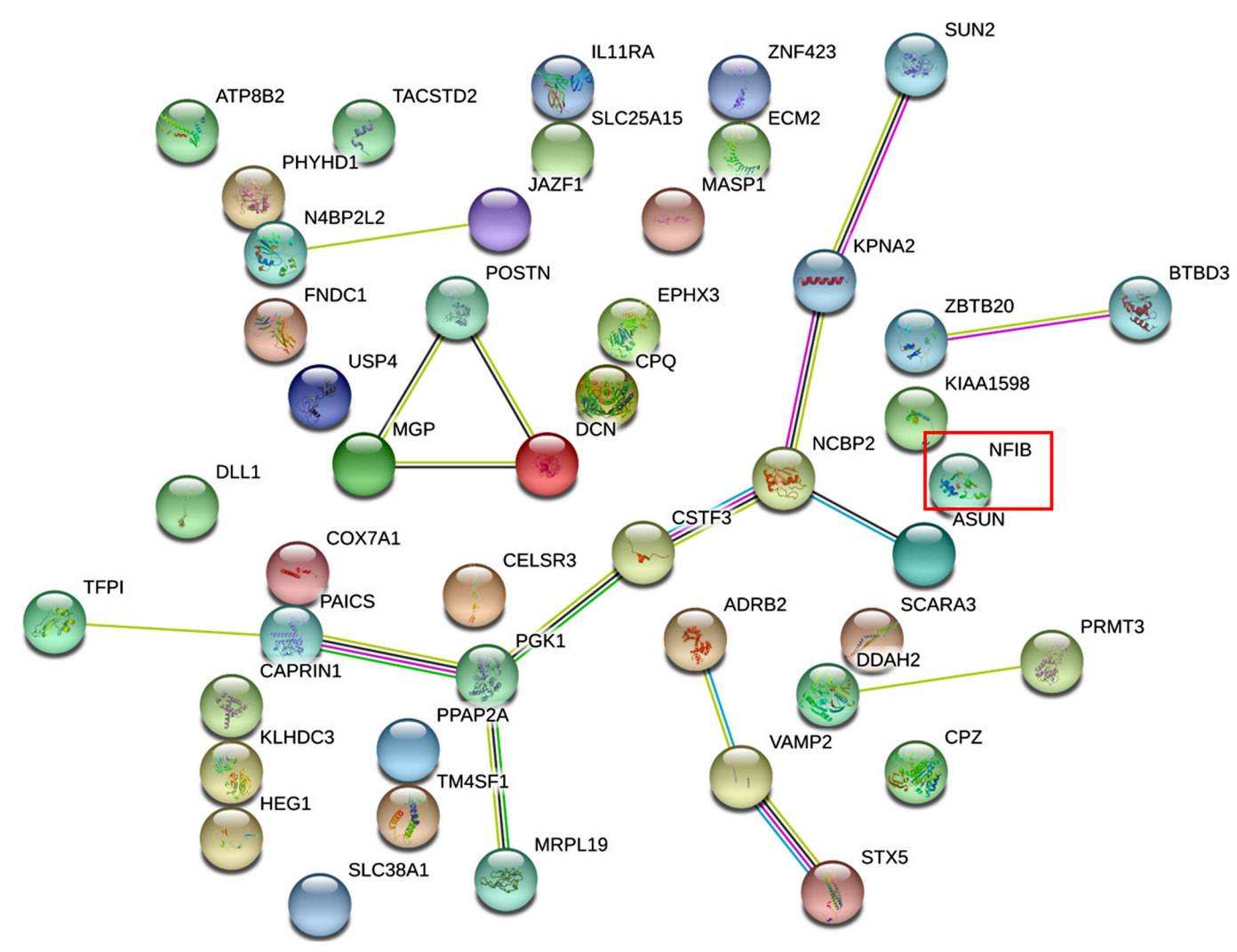

Figure 2. Protein-protein interaction network of the 46 differentially expressed genes in human papilloma virus-independent vulvar squamous cell carcinoma (constructed with STRING). nuclear factor IB is indicated with a red box. Network nodes represent all the proteins produced by a single, protein-coding gene locus. Colored nodes represent query proteins and first shells of interactions. White nodes represent second shell of interactions. Empty nodes represent proteins of an unknown 3D structure. Filled nodes represent proteins of which some 3D structures are known or predicted. The edges are coded as follows: Light blue, known interaction curated from databases; pink, known interaction determined through experiments; green, predicted interaction in the gene neighborhood; red, gene fusions; dark blue, gene co-occurrence; lime green, text mining; black, co-expression; indigo, protein homology.

HSIL ( $n=6$ ): All showed strong nuclear NFIB-expression along the basal layers and occasionally in the para-basal layers. Staining in the basal layer was discontinuous, and expression in the para-basal layers was primarily seen only at the tips of rete ridges.

HPV-related VSCC $(n=5)$ : Two were completely negative, and 2 were predominantly negative, showing only focal, weak, nuclear NFIB-expression along the periphery of the tumor cell nests. One VSCC showed NFIB-expression of moderate intensity along the periphery of the tumor cell nests.

dVIN ( $n=6)$ : One dVIN was completely negative and 5 showed only focal, weak, nuclear NFIB-expression.

HPV-independent VSCC $(n=6)$ : One was completely negative, and 5 were predominantly negative, showing only focal, 
Table III. GO enrichment analysis of differentially expressed genes for human papilloma virus-independent vulvar squamous cell carcinoma.

A, Biological processes

\begin{tabular}{lllr}
\hline Term & \multicolumn{1}{c}{ Description } & Gene count & P-value \\
\hline GO:0050794 & Regulation of cellular process & 33 & $5.27 \times 10^{-12}$ \\
GO:0008152 & Metabolic process & 31 & $1.02 \times 10^{-11}$ \\
GO:0016043 & Cellular component organization & 25 & $7.37 \times 10^{-09}$ \\
GO:0032502 & Developmental process & 22 & $3.69 \times 10^{-11}$ \\
GO:0032501 & Multicellular organismal process & 19 & $4.55 \times 10^{-11}$ \\
GO:0065008 & Regulation of biological quality & 19 & $6.93 \times 10^{-12}$ \\
GO:0007275 & Multicellular organism development & 19 & $7.01 \times 10^{-08}$ \\
GO:0007154 & Cell communication & 18 & 18 \\
GO:0030154 & Cell differentiation & 17 & $1.04 \times 10^{-06}$ \\
GO:0042221 & Transport & 17 & $8.87 \times 10^{-10}$ \\
\hline
\end{tabular}

B, Molecular functions

\begin{tabular}{llrr}
\hline Term & \multicolumn{1}{c}{ Description } & Gene count & P-value \\
\hline GO:0005515 & Protein binding & 38 & $5.43 \times 10^{-07}$ \\
GO:0043167 & Ion binding & 20 & $7.70 \times 10^{-10}$ \\
GO:0003824 & Catalytic activity & 13 & $1.00 \times 10^{-11}$ \\
GO:0097159 & Organic cyclic compound binding & 12 & $7.47 \times 10^{-09}$ \\
GO: 1901363 & Heterocyclic compound binding & 11 & $8.24 \times 10^{-09}$ \\
GO:0003676 & Nucleic acid binding & 8 & 7 \\
GO:0016787 & Hydrolase activity & 7 & $5.53 \times 10^{-05}$ \\
GO:0043168 & Anion binding & 7 & $8.30 \times 10^{-12}$ \\
GO:0098772 & Molecular function regulator & 6 & $7.05 \times 10^{-06}$ \\
GO:0097367 & Carbohydrate derivative binding & $6.35 \times 10^{-05}$ \\
\hline
\end{tabular}

C, Cellular component

\begin{tabular}{lllr}
\hline Term & \multicolumn{1}{c}{ Description } & Gene count & P-value \\
\hline GO:0043227 & Membrane-bounded organelle & 36 & $2.20 \times 10^{-09}$ \\
GO:0005737 & Cytoplasm & 31 & $1.82 \times 10^{-09}$ \\
GO:0016020 & Cell membrane & 26 & $2.41 \times 10^{-11}$ \\
GO:0005576 & Extracellular region & 20 & $7.74 \times 10^{-05}$ \\
GO:0031224 & Intrinsic component of membrane & 20 & $3.26 \times 10^{-06}$ \\
GO:0005634 & Nucleus & 18 & $7.60 \times 10^{-11}$ \\
GO:0031982 & Vesicle & 14 & $5.76 \times 10^{-05}$ \\
GO:0071944 & Cell periphery & 14 & $7.46 \times 10^{-07}$ \\
GO:0043233 & Organelle lumen & 14 & $8.72 \times 10^{-12}$ \\
GO:0012505 & Endomembrane system & 13 & $3.75 \times 10^{-10}$ \\
\hline
\end{tabular}

GO, Gene Ontology.

weak, nuclear NFIB-expression along the periphery of the tumor cell nests.

Immunohistochemical expressions of p53, p16, STAT1, and NFIB in normal vulvar tissue, HSIL, dVIN, and VSCC (both subtypes) are demonstrated in Figs. 3-7.

\section{Discussion}

In this study, we utilized bioinformatics tools to gain insight into VSCC carcinogenesis, and to identify potential biomarkers that may have diagnostic, prognostic, or therapeutic 
Table IV. Functional annotation analysis of differentially expressed genes for human papilloma virus-independent vulvar squamous cell carcinoma.

\begin{tabular}{lcr}
\hline Functional annotation cluster & Gene count & P-value \\
\hline Developmental protein & 21 & $5 \times 10^{-07}$ \\
Calcium ion binding & 16 & $2 \times 10^{-05}$ \\
EGF-like domain & 12 & $1 \times 10^{-04}$ \\
EGF-like calcium-binding, conserved site & 11 & $7 \times 10^{-06}$ \\
Cell-cell adhesion & 7 & $2 \times 10^{-04}$ \\
Integral component of membrane & $6 \times 10^{-05}$ \\
Cell differentiation & 5 & $1 \times 10^{-04}$ \\
Acetylation & $1 \times 10^{-05}$ \\
Extracellular matrix organization & 4 & $2 \times 10^{-05}$ \\
Transmembrane helix & 3 & $6 \times 10^{-04}$ \\
& 3 & \\
\hline
\end{tabular}

Table V. Immonohistochemical expression patterns of p53 and p16.

\begin{tabular}{|c|c|c|c|c|c|}
\hline \multirow[b]{2}{*}{ Marker and expression pattern } & \multicolumn{5}{|c|}{ Diagnosis } \\
\hline & $\begin{array}{l}\text { Normal vulvar tissue } \\
\qquad(\mathrm{n}=7)\end{array}$ & $\begin{array}{l}\text { HSIL } \\
(n=6)\end{array}$ & $\begin{array}{l}\text { HPV-related } \\
\text { VSCC }(n=5)\end{array}$ & $\begin{array}{l}\mathrm{dVIN} \\
(\mathrm{n}=6)\end{array}$ & $\begin{array}{l}\text { HPV-independent } \\
\text { VSCC }(n=6)\end{array}$ \\
\hline \multicolumn{6}{|l|}{ p53-mut } \\
\hline Parabasal/diffuse & $0(0)$ & $0(0)$ & $1(20)$ & $5(83)$ & $3(50)$ \\
\hline Basal & $0(0)$ & $0(0)$ & $0(0)$ & $0(0)$ & $0(0)$ \\
\hline Absent/null & $0(0)$ & $0(0)$ & $0(0)$ & $0(0)$ & $2(33)$ \\
\hline \multicolumn{6}{|l|}{ p53-wt } \\
\hline Wild-type scattered & $7(100)$ & $0(0)$ & $0(0)$ & $1(17)$ & $1(17)$ \\
\hline Wild-type mid-epithelial & $0(0)$ & $6(100)$ & $4(80)$ & $0(0)$ & $0(0)$ \\
\hline \multicolumn{6}{|l|}{ 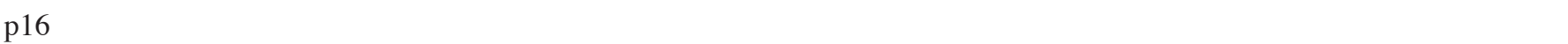 } \\
\hline Block-type & $0(0)$ & $6(100)$ & $5(100)$ & $0(0)$ & $0(0)$ \\
\hline Non-block-type/patchy & $0(0)$ & $0(0)$ & $0(0)$ & $1(17)$ & $0(0)$ \\
\hline No expression & $7(100)$ & $0(0)$ & $0(0)$ & $5(83)$ & $6(100)$ \\
\hline
\end{tabular}

Data are presented as n (\%). HSIL, high grade squamous intraepithelial lesions; HPV, human papilloma virus; VSCC, vulvar squamous cell carcinoma; dVIN, differentiated vulvar intraepithelial neoplasias; mut, mutant; wt, wild-type.

Table VI. Immunohistochemical expression of NFIB and STAT1.

\section{Diagnosis}

\begin{tabular}{lccccc}
\cline { 2 - 6 } $\begin{array}{l}\text { Immunohistochemical } \\
\text { marker }\end{array}$ & $\begin{array}{c}\text { Normal vulvar } \\
\text { tissue }(\mathrm{n}=7)\end{array}$ & HSIL $(\mathrm{n}=6)$ & $\begin{array}{c}\text { HPV-related } \\
\text { VSCC }(\mathrm{n}=5)\end{array}$ & $\mathrm{dVIN}(\mathrm{n}=6)$ & $\begin{array}{c}\text { HPV-independent VSCC } \\
(\mathrm{n}=6)\end{array}$ \\
\hline NFIB & $18(10.3-23.1)$ & $12.5(9.1-17.6)$ & $5(1.1-9.2)$ & $6(4.3-10.1)$ & $2.5(0.9-11.3)$ \\
STAT1 & $65(50.8-87.5)$ & $67.5(38.8-81.2)$ & $80(68.5-91.5)$ & $85(69.1-92.5)$ & $90(53.1-95.6)$
\end{tabular}

Data are presented as the mean 95\% confidence interval. NFIB, nuclear factor IB; STAT1, signal transducer and activator of transcription 1; HSIL, high grade squamous intraepithelial lesions; HPV, human papilloma virus; VSCC, vulvar squamous cell carcinoma; dVIN, differentiated vulvar intraepithelial neoplasias.

applications. For both subtypes of VSCC (i.e., HPV-related and HPV-independent) we identified a set of DEGs that appeared to be similarly regulated (up or down) in two independent gene expression microarray datasets. We found that the majority of DEGs that were identified for HPV-related VSCC are involved in the immune response, whereas those identified for 
A

B

C

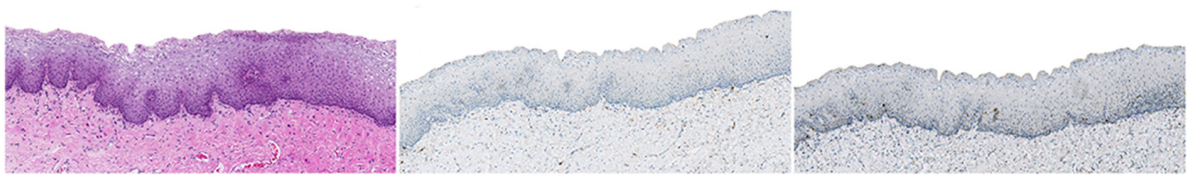

D

E

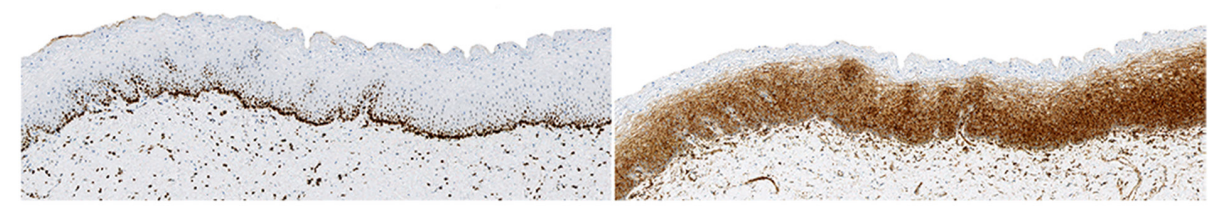

Figure 3. Normal vulvar tissue histology and IHC. (A) Histological appearance (hematoxylin and eosin stain). (B) p16-IHC was negative. (C) p53-IHC revealed wild-type expression. (D) NFIB-IHC exhibited strong, diffuse, nuclear expression, predominantly along the basal layers and occasionally in the para-basal layers. (E) signal transducer and activator of transcription 1-IHC demonstrated diffuse, cytoplasmic expression of moderate-to-strong intensity, across full epithelial thickness (A-C, magnification, x100; D and E, magnification, x200). IHC, immunohistochemistry.

A

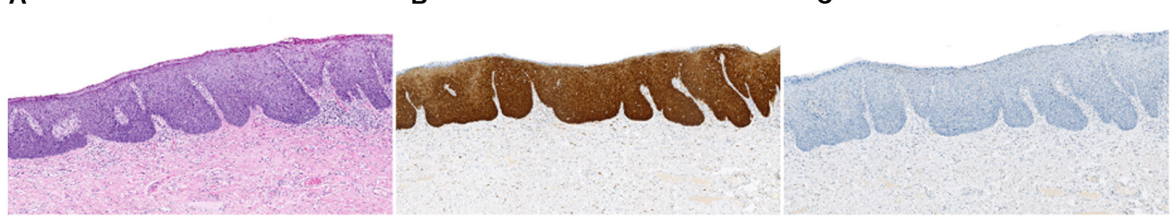

D

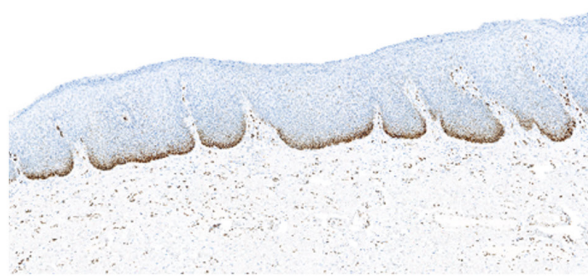

$\mathrm{E}$

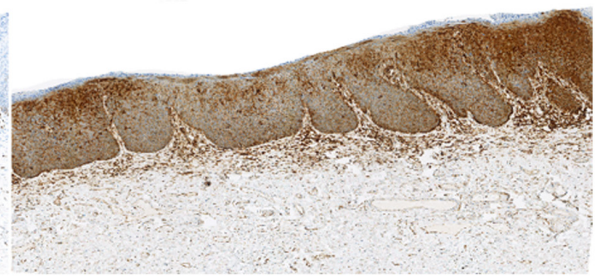

Figure 4. High grade squamous intraepithelial lesion histology and IHC. (A) Histological appearance (hematoxylin and eosin staining). (B) p16-IHC demonstrated block-type expression. (C) p53-IHC exhibited wild-type expression. (D) Nuclear factor IB-IHC demonstrated strong nuclear expression along the basal layers and occasionally in the parabasal layers. Staining in the basal layer was discontinuous, and expression in the parabasal layers was mainly limited to the tips of rete ridges. (E) Signal transducer and activator of transcription 1-IHC revealed diffuse, cytoplasmic expression of moderate-to-strong intensity, across full epithelial thickness (A-C, magnification, x100; D and E, magnification, x200). IHC, immunohistochemistry.

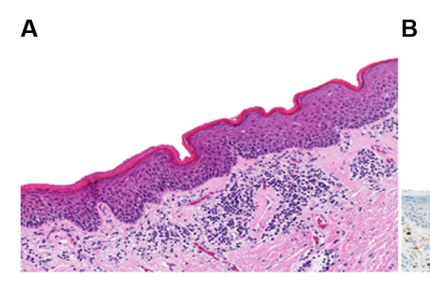

D

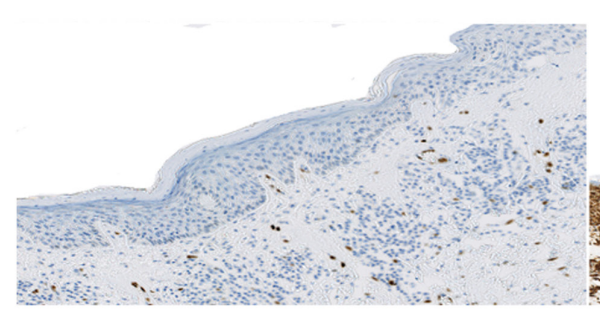

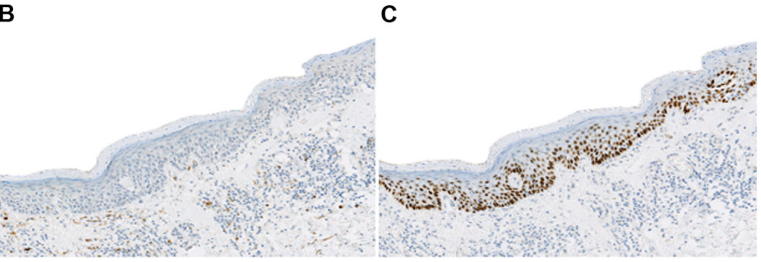

$\mathrm{E}$

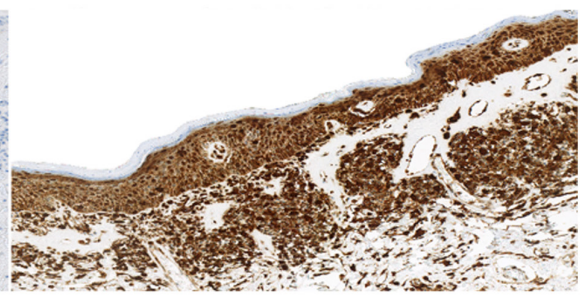

Figure 5. Differentiated vulvar intraepithelial neoplasia histology and IHC. (A) Histological appearance (HE-stain). (B) p16-IHC is completely negative. (C) p53-IHC shows mutation-pattern. (D) nuclear factor IB-IHC is completely negative. (E) signal transducer and activator of transcription 1-IHC shows diffuse, cytoplasmic expression of moderate-to-strong intensity, across full epithelial thickness (A-C, original magnification, x100; D and E, original magnification, x200). IHC, immunohistochemistry. 


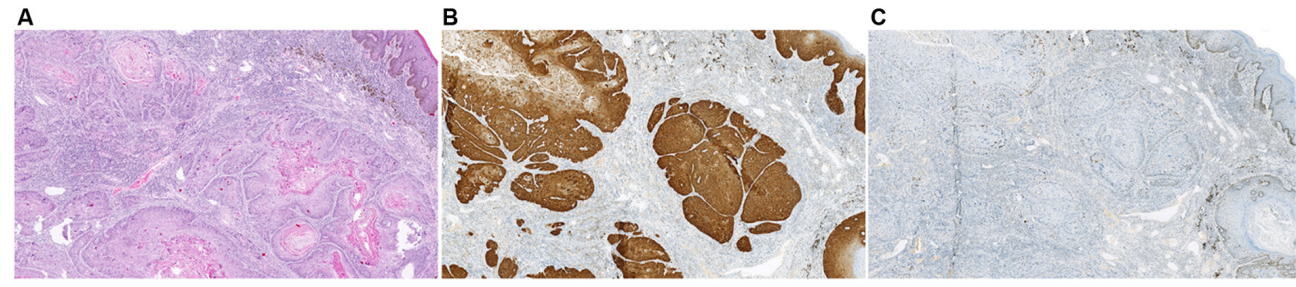

D

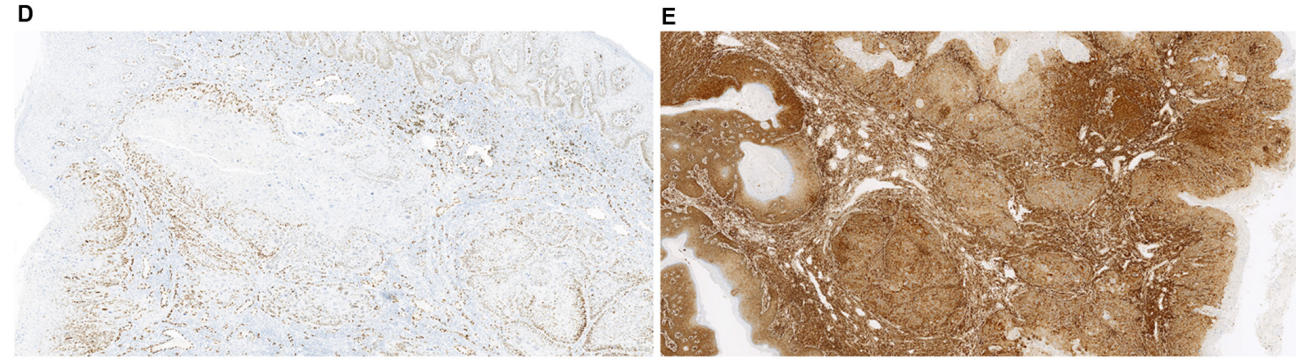

Figure 6. Human papillomavirus related vulvar squamous cell carcinoma histology and IHC. (A) Histological appearance (hematoxylin and eosin staining). (B) p16-IHC demonstrated block-type expression. (C) p53-IHC demonstrated wild-type expression. (D) NFIB-IHC was negative in certain tumor nests, demonstrating focal, weak, nuclear expression along the periphery of some tumor nests. (E) STAT1-IHC exhibited diffuse, cytoplasmic expression of moderate-to-strong intensity, across full epithelial thickness (A-C, magnification, x100; D and E, magnification, x200). IHC, immunohistochemistry; NFIB, nuclear factor IB; STAT1, signal transducer and activator of transcription 1.

A

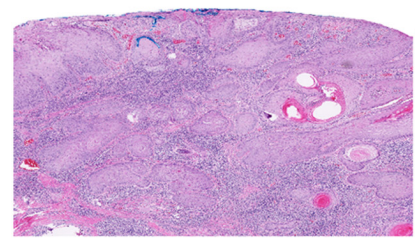

D

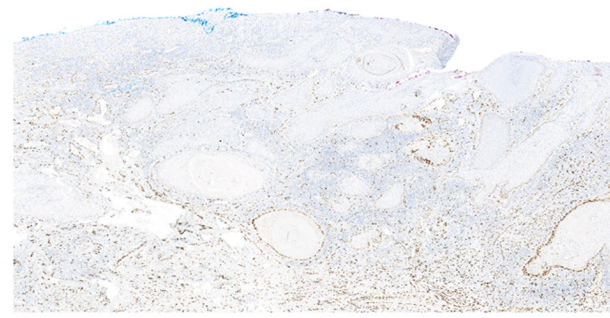

B

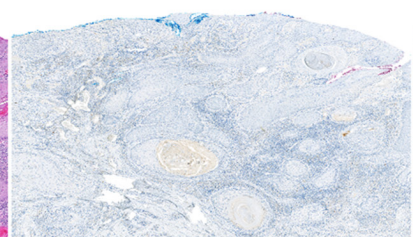

E

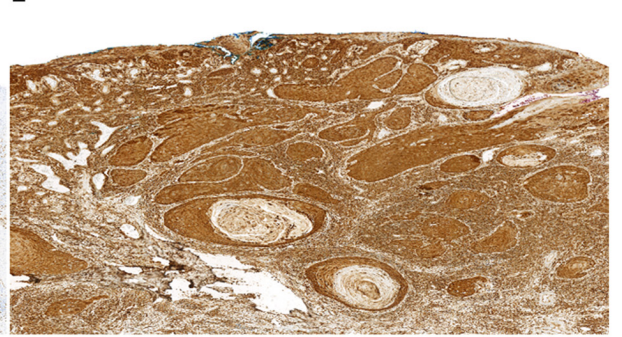

Figure 7. Human papillomavirus independent vulvar squamous cell carcinoma: Histology and IHC. (A) Histological appearance (hematoxylin and eosin staining). (B) p16-IHC was completely negative. (C) p53-IHC demonstrated a mutation-pattern. (D) NFIB-IHC was negative in certain tumor nests, demonstrating focal, weak, nuclear expression along the periphery of some of the tumor nests. (E) STAT1-IHC revealed diffuse, cytoplasmic expression of moderate-to-strong intensity, across full epithelial thickness (A-C, magnification, x100; D and E, magnification, x200). IHC, immunohistochemistry; NFIB, nuclear factor IB; STAT1, signal transducer and activator of transcription 1.

HPV-independent VSCC were involved in second messenger signaling-this provides support for the dual pathogenesis of VSCC.

We studied the expression of two of the DEGs, i.e. NFIB and STAT1, that were found to be similarly regulated in both datasets, in whole tissue sections of VSCCs, dVINs, HSILs, and normal vulvar tissues, by performing IHC. NFIB was identified to be downregulated in HPV-independent VSCC, and STAT1 was identified to be upregulated in HPV-related VSCC. Neither of these markers has been previously studied for VSCC or its precursor lesions.

NFIB showed strong, nuclear expression in the basal and para-basal epithelial layers in normal vulvar tissue, whereas, in dVIN and both subtypes of VSCC, NFIB was either completely negative or minimally expressed. NFIB expression was also reduced in HSIL in comparison with normal vulvar tissue, but to a lesser extent than that in dVIN and VSCC.

NFIB is a transcription factor which has tumor suppressive, as well as, oncogenic potential (23). In cervical SCC and head-and-neck SCC (HNSCC), NFIB-expression has been observed to be lower than in normal tissues from the corresponding sites (23). Furthermore, lower levels of NFIB-expression have been reported to correlate significantly with worse prognosis for both of these malignancies (23). Interestingly, NFIB is a key regulator of the aryl hydrocarbon pathway, which we previously identified to be involved in HPV-independent VSCC (2). In addition, high-confidence proximity interactions have been reported between NFIB and 
SOX2 (24); SOX2 is a cancer-stemness related transcription factor that is overexpressed in dVIN and VSCC (25). In view of these observations, we believe that the role of NFIB in VSCC and its potential as a therapeutic target deserve further investigation. In addition to SCCs, genomic alterations of NFIB have been detected in several other malignancies, as shown in Fig. S2.

Unlike NFIB, no discernable difference was observed in immunohistochemical expression of STAT1 between normal vulvar tissue, dVIN, HSIL, or VSCC (both subtypes). For all tissue types, diffuse, cytoplasmic STAT1-expression of moderate-to-strong intensity was noted across full epithelial thickness. STAT1 is a component of the Janus kinase (JAK)-STAT signaling pathway, and can act as an antimicrobial mediator, a tumor suppressor, or a promotor of tumor progression (26). Aberrant expression of STAT1 in HPV-related lesions is considered to reflect activation of the JAK-STAT pathway as a consequence of the inflammatory response induced by HPV (26).

Our results regarding IHC-expression of STAT1 were in contrast to those of a recent study, which reported a higher STAT1-expression in cervical intraepithelial neoplasia (CIN) than in normal cervical epithelium, and deduced an association of increased STAT1-expression with malignant progression of CIN (26). Since STAT-1 expression is regulated by a complex network of interferons, we speculate that the difference in expression between vulvar and cervical tissue could be ascribed to the dissimilar microenvironments of these anatomical sites. Similarly to NFIB, genomic alterations of STAT1 have been detected in several malignancies, as shown in Fig. S3.

This study was an attempt to leverage bioinformatics to identify DEGs in VSCC. We identified NFIB as a downregulated gene in VSCC, and observed that its immunohistochemical expression was reduced in both subtypes of VSCC. Hence, we believe that the relevance of NFIB as a diagnostic/prognostic biomarker deserves further exploration. However, an apparent limitation of this study is that the DEGs were identified from datasets consisting of small sample sizes, and IHC was also performed on a limited set of tissues. Further experiments are needed to confirm the function of these DEGs in VSCC and to validate their immunohistochemical expression in vulvar tissues.

Nevertheless, we hope that our results will instigate further research into VSCC carcinogenesis and pave the path for unravelling novel biomarkers of VSCC and its precursor lesions.

\section{Acknowledgements}

The authors would like to thank Dr Leen J. Blok (Erasmus $\mathrm{MC}$ ) for the use of data on gene expression analysis.

\section{Funding}

No funding was received.

\section{Availability of data and materials}

The datasets used and/or analyzed during the current study are available from the corresponding author on reasonable request.

\section{Authors' contributions}

SD, PCEG, TPPVDB, SMAS, LAMS, PJVDS, SK and FJVK conceived and designed the methodology of the current study. SMAS, PJVDS analyzed the data. SD, SMAS, PJVDS and FJVK confirm the authenticity of all the raw data. TPPVDB and LAMS performed the experiments. SD, PCEG, TPPVDB, SS, LAMS, HCVD and PJVDS curated the pathological and clinical data. SD drafted the manuscript, constructed the graphs and arranged the histology figures. PCEG, SK and FJVK supervised the current study. All authors reviewed and edited the manuscript and read and approved the final manuscript.

\section{Ethics approval and consent to participate}

The current study was conducted in accordance with the guidelines of the Dutch Federation of Biomedical Scientific Societies (www.federa.org/codes-conduct), which state that no separate ethical approval is required for the use of anonymized residual tissue procured during regular treatment. This is also part of the standard treatment agreement at Erasmus MC.

\section{Patient consent for publication}

Not applicable.

\section{Competing interests}

The authors declare that they have no competing interests.

\section{References}

1. Prieske K, Alawi M, Oliveira-Ferrer L, Jaeger A, Eylmann K, Burandt E, Schmalfeldt B, Joosse SA and Woelber L: Genomic characterization of vulvar squamous cell carcinoma. Gynecol Oncol 158: 547-554, 2020.

2. Dasgupta S, Ewing-Graham PC, Swagemakers SMA, van der Spek PJ, van Doorn HC, Noordhoek Hegt V, Koljenović S and van Kemenade FJ: Precursor lesions of vulvar squamous cell carcinoma-histology and biomarkers: A systematic review. Crit Rev Oncol Hematol 147: 102866, 2020.

3. de Martel C, Georges D, Bray F, Ferlay J and Clifford GM: Global burden of cancer attributable to infections in 2018: A worldwide incidence analysis. Lancet Glob Health 8: e180-e190, 2020.

4. Zeimet AG: Molecular characterization of vulvar squamous cell cancer: High time to gain ground. Gynecol Oncol 158: 519-520, 2020.

5. Han MR, Shin S, Park HC, Kim MS, Lee SH, Jung SH, Song SY, Lee SH and Chung YJ: Mutational signatures and chromosome alteration profiles of squamous cell carcinomas of the vulva. Exp Mol Med 50: e442, 2018.

6. Weberpals JI, Lo B, Duciaume MM, Spaans JN, Clancy AA, Dimitroulakos J, Goss GD and Sekhon HS: Vulvar squamous cell carcinoma (VSCC) as two diseases: HPV status identifies distinct mutational profiles including oncogenic fibroblast growth factor receptor 3. Clin Cancer Res 23: 4501-4510, 2017.

7. Nooij LS, Ter Haar NT, Ruano D, Rakislova N, van Wezel T, Smit VTHBM, Trimbos BJBMZ, Ordi J, van Poelgeest MIE and Bosse T: Genomic characterization of vulvar (Pre)cancers identifies distinct molecular subtypes with prognostic significance. Clin Cancer Res 23: 6781-6789, 2017.

8. Zieba S, Pouwer AW, Kowalik A, Zalewski K, Rusetska N, Bakuła-Zalewska E, Kopczyński J, Pijnenborg JMA, de Hullu JA and Kowalewska M: Somatic mutation profiling in premalignant lesions of vulvar squamous cell carcinoma. Int J Mol Sci 21: 4880, 2020 . 
9. Williams EA, Werth AJ, Sharaf R, Montesion M, Sokol ES, Pavlick DC, McLaughlin-Drubin M, Erlich R, Toma H, Williams KJ, et al: Vulvar squamous cell carcinoma: Comprehensive genomic profling of $\mathrm{HPV}^{+}$versus $\mathrm{HPV}$-forms reveals distinct sets of potentially actionable molecular targets. JCO Precis Oncol 4: 647-661, 2020.

10. Tessier-Cloutier B, Kortekaas KE, Thompson E, Pors J, Chen J, Ho J, Prentice LM, McConechy MK, Chow C, Proctor L, et al: Major p53 immunohistochemical patterns in in situ and invasive squamous cell carcinomas of the vulva and correlation with TP53 mutation status. Mod Pathol 33: 1595-1605, 2020.

11. Tessier-Cloutier B, Pors J, Thompson E, Ho J, Prentice L, McConechy M, Aguirre-Hernandez R, Miller R, Leung S, Proctor L, et al: Molecular characterization of invasive and in situ squamous neoplasia of the vulva and implications for morphologic diagnosis and outcome. Mod Pathol 34: 508-518, 2021.

12. Ge Y, Zhang C, Xiao S, Liang L, Liao S, Xiang Y, Cao K, Chen H and Zhou Y: Identification of differentially expressed genes in cervical cancer by bioinformatics analysis. Oncol Lett 16 : 2549-2558, 2018

13. Micci F, Panagopoulos I, Haugom L, Dahlback HS, Pretorius ME, Davidson B, Abeler VM, Tropé CG, Danielsen HE and Heim S: Genomic aberration patterns and expression profiles of squamous cell carcinomas of the vulva. Genes Chromosomes Cancer 52: $551-563,2013$

14. Huang da W, Sherman BT and Lempicki RA: Systematic and integrative analysis of large gene lists using DAVID bioinformatics resources. Nat Protoc 4: 44-57, 2009.

15. Huang DW, Sherman BT, Tan Q, Kir J, Liu D, Bryant D, Guo Y, Stephens R, Baseler MW, Lane HC and Lempicki RA: DAVID bioinformatics resources: Expanded annotation database and novel algorithms to better extract biology from large gene lists. Nucleic Acids Res 35 (Web Server Issue): W169-W175, 2007.

16. Cerami E, Gao J, Dogrusoz U, Gross BE, Sumer SO, Aksoy BA, Jacobsen A, Byrne CJ, Heuer ML, Larsson E, et al: The cBio cancer genomics portal: An open platform for exploring multidimensional cancer genomics data. Cancer Discov 2: 401-404, 2012.

17. Gao J, Aksoy BA, Dogrusoz U, Dresdner G, Gross B, Sumer SO, Sun Y, Jacobsen A, Sinha R, Larsson E, et al: Integrative analysis of complex cancer genomics and clinical profiles using the cBioPortal. Sci Signal 6: pl1, 2013.

18. Szklarczyk D, Gable AL, Lyon D, Junge A, Wyder S, Huerta-Cepas J, Simonovic M, Doncheva NT, Morris JH, Bork P, et al: STRING v11: Protein-protein association networks with increased coverage, supporting functional discovery in genome-wide experimental datasets. Nucleic Acids Res 47D D607-D613, 2019.
19. Tang Z, Li C, Kang B, Gao G, Li C and Zhang Z: GEPIA: A web server for cancer and normal gene expression profiling and interactive analyses. Nucleic Acids Res 45W: W98-W102, 2017.

20. Darragh TM, Colgan TJ, Cox JT, Heller DS, Henry MR, Luff RD, McCalmont T, Nayar R, Palefsky JM, Stoler MH, et al: The lower anogenital squamous terminology standardization project for HPV-associated lesions: Background and consensus recommendations from the college of American pathologists and the American society for colposcopy and cervical pathology. Arch Pathol Lab Med 136: 1266-1297, 2012.

21. Kortekaas KE, Solleveld-Westerink N, Tessier-Cloutier B, Rutten TA, Poelgeest MIE, Gilks CB, Hoang LN and Bosse T: Performance of the pattern-based interpretation of p53 immunohistochemistry as a surrogate for TP53 mutations in vulvar squamous cell carcinoma. Histopathology 77: 92-99, 2020.

22. Heller DS, Day T, Allbritton JI, Scurry J, Radici G, Welch K and Preti M; ISSVD Difficult Pathologic Diagnoses Committee: Diagnostic criteria for differentiated vulvar intraepithelial neoplasia and vulvar aberrant maturation. J Low Genit Tract Dis 25: 57-70, 2021.

23. Li Y, Sun C, Tan Y, Li L, Zhang H, Liang Y, Zeng J and Zou H: Transcription levels and prognostic significance of the NFI family members in human cancers. PeerJ 8: e8816, 2020.

24. Kim BR, Coyaud E, Laurent EMN, St-Germain J, Van de Laar E, Tsao MS, Raught B and Moghal N: Identification of the SOX2 interactome by BioID reveals EP300 as a mediator of SOX2-dependent squamous differentiation and lung squamous cell carcinoma growth. Mol Cell Proteomics 16: 1864-1888, 2017.

25. Brustmann $\mathrm{H}$ and Brunner A: Immunohistochemical expression of SOX2 in vulvar intraepithelial neoplasia and squamous cell carcinoma. Int J Gynecol Pathol 32: 323-328, 2013.

26. Wu S, Wu Y, Lu Y, Yue Y, Cui C, Yu M, Wang S, Liu M, Zhao Y and Sun Z: STAT1 expression and HPV16 viral load predict cervical lesion progression. Oncol Lett 20: 28, 2020. 